International Journal of Modern Physics C

(C) World Scientific Publishing Company

\title{
Orbital carriers and inheritance in discrete-time quadratic dynamics
}

\author{
Jason A.C. Gallas \\ Instituto de Altos Estudos da Paraíba, Rua Silvino Lopes 419-2502, \\ 58039-190 João Pessoa, Brazil, \\ Complexity Sciences Center, 9225 Collins Ave. Suite 1208, Surfside FL 33154, USA, \\ Max-Planck-Institut für Physik komplexer Systeme, 01187 Dresden, Germany \\ jason.gallas@gmail.com
}

Received 2 March 2020

Accepted 24 March 2020

Published 22June 2020

https://doi.org/10.1142/S0129183120501004

\begin{abstract}
Explicit formulas for orbital carriers of periods 4, 5, and 6 are reported for discrete-time quadratic dynamics. A systematic investigation of orbital inheritance for periods as high as $k \leq 12$ is also reported. Inheritance means that unknown orbits may be obtained by nonlinear transformations of known orbits. Such nested orbit within orbit stratification shows orbits not to be necessarily independent of each other as generally assumed. Orbital stratification is potentially significant to rearrange trajectories sums in trace formulas underlying modern semiclassical interpretations of atomic physics spectra. The stratification seems to dominate as the orbital period grows.
\end{abstract}

Keywords: Orbital carriers; Orbital inheritance; Quadratic dynamics; Symbolic computation.

PACS Nos.: 02.70.Wz, 02.10.De, 03.65.Fd

\section{Introduction}

Applied problems in physics normally require solving equations of motion, frequently expressed either as differential equations involving continuous-time derivatives, or discrete-time maps. Since the advent of modern computers, solving equations of motion essentially boils down to number crunching using special-purpose numerical methods. For a representative selection of methods and applications see, e.g., Refs. 1213.

Numerical methods revealed much of what is presently known about the time-evolution of complex systems. However, there are certain peculiarities that are totally out of reach to approximate numerical methods and that have not yet been addressed as they could. For instance, consider cascades of periodic motions, which are among the most prominent features found in systems governed by differential equations or by maps. Although such cascades cannot be followed analytically for differential equations, they are accessible in systems governed by maps with algebraic equations of motion, particularly in one-dimensional dissipative maps $\frac{122 \mid 3}{1}$.

Consider a popular class of models, namely one-dimensional maps governed by algebraic equations of motion. To delimit analytically their stability windows one needs to solve polynomials containing physical parameters. Although parameters may vary freely, conditions imposed on the stability boundaries greatly reduce such freedom as well as the complexity of the numerical values defining boundaries. For example, for the paradigmatic quadratic and Hénon maps, the polynomial coefficients at intersections are simply given by integers or by algebraic numbers $\$ 12|3| 4 \mid 5$.

The self-similar regularities recorded for cascades of periodic motions in parameterized maps 
pose a natural question regarding the generic arithmetic nature of the numbers delimiting adjacent windows of stability as parameters are varied. Knowledge of the arithmetical unfolding of such cascades should provide insight into the analytical clockwork mechanism underlying this forever repeating process. Such information cannot be inferred from approximate computations but could be eventually won using exact algebraic analysis.

The purpose of this paper is to report a systematic investigation of orbital carriers and orbital inheritance in discrete-time quadratic dynamics, in the so-called partition generating limit $\underline{112}$, whose equation of motion is $x_{t+1}=2-x_{t}^{2}$. More specifically, we extend previous work ${ }^{5}$ to include carriers for orbits with periods 4,5 and 6 , and inheritance for periods $k \leq 12$ of the map. Such computations are quite strenuous. The latter limit is set by the capability of the hardware and software at our disposal to generate and to factor large polynomials of degrees no less than 4020, with exceedingly large numerical coefficients and discriminants. As discussed and illustrated below, carriers are polynomials encoding simultaneously all possible orbits of a given period ${ }^{5}$. Inheritance means that known periodic orbits reveal unknown orbits. New orbits are obtained through simple nonlinear transformations from known orbits $6 / 7 / 8$. Inherited orbits are clones that share an arithmetic ancestry. Arithmetic interdependencies among periodic orbits are hard, not to say impossible, to recognize in numerical simulations, where only approximate numbers are considered.

The starting point to investigate the arithmetic nature of equations of motion is the ring $\mathbb{Z}$ of integers, namely solving polynomials with integer coefficients. Key properties which facilitate the study of polynomials with integer coefficients are the Euclidean algorithm and the unique factorization of integers (the 'fundamental theorem of arithmetic'). Such properties no longer always hold for rings of integers of higher algebraic number fields, involving polynomials with a good deal more complicated coefficients and which are the framework where algebraic equations of motions must be considered.

The first coherent discussion of complex integers $a+i b$ with rational integral $a$ and $b$ was presented by Gauss as far back as 1831-32, in his second paper on biquadratic reciprocity. Subsequently, the theory of quadratic algebraic numbers was essentially completed during the nineteen century by Kummer, Dirichlet, Dedekind, Hilbert and others9. However, the corresponding knowledge regarding numbers as simple as cubics and relative cubics is by far less complete, despite more than two centuries of work 10 . The main difficulty comes from the wellknown fact that irreducible cubics with three real roots, the so-called casus irreducibilis, cannot have their roots expressed in terms of real radicals. The equations of motion discussed here are attractive in that they require investigating towers of such cubic fields. We consider periods $k \leq 12$, and provide explicit solutions for polynomials of degrees as high as 18 and 24, involving nested cubic roots.

With respect to applications beyond the scope of dynamical systems, we mention briefly that the concept of inheritance is potentially attractive for atomic physics, where it seems to imply the interesting and unsuspected possibility of rearranging certain orbit-dependent contributions in cycle expansions and semiclassical sums needed for calculating energy spectra and density of states using, e.g., Gutzwiller's trace formula $15|16| 17|18| 19|20| 21 \mid 22$.

\section{Orbital carriers for periods 4,5 and 6}

A recent work has shown that classical equations of motion of algebraic origin may be all conveniently extracted from just a single mathematical object, a polynomial called an orbital carrier. All possible orbits may be encoded simultaneously by a single carrier, with individual orbits parameterized by $\sigma$, the sum of their orbital points ${ }^{5}$. In Ref. ${ }^{5}$, such parameterization was 
established for period-three orbits using standard textbook knowledge of the theory of algebraic equations. Essentially, one uses certain functions of the roots of the equation of motion, the elementary symmetric functions ${ }^{23}$, which may be expressed in a general manner by means of the coefficients of the equation of motion, without the equation itself being resolved. This fact shifts the traditional study of orbital points to a new level, to the study of orbital equations of motion.

Here, we extend the aforementioned orbital parameterization to include explicit expressions for carriers of periods 4,5 and 6 . Results for periods four 24 and six 25 may be obtained as particular cases of general expressions obtained previously for the two-parameter Hénon map, $(x, y) \mapsto\left(a-x^{2}+b y, x\right)$. For arbitrary values of $a$, carriers for the quadratic map $x_{t+1}=a-x_{t}^{2}$ are obtained setting $b=0$ in the expressions of the Hénon map. For the partition generating limit discussed here, set $(a, b)=(2,0)$. The carrier for period five is freshly obtained and is reported here for the first time. Apart from the theoretical novelty of these carriers, they help to motivate the main results below and to make them more comprehensible.

\subsection{The period four carrier}

Essentially, for a given period $k$, all period- $k$ orbits may be encoded simultaneously by two polynomials, as described in a recent open access paper ${ }^{5}$ : A $\sigma$-parameterized polynomial $\psi_{k}(x)$, called the carrier, and an auxiliary polynomial, $\mathbb{S}_{k}(\sigma)$, which fixes the values of the parameter $\sigma$ for each individual orbit. The parameter $\sigma$ is just the sum of the orbital points. The degree of the polynomial $\mathbb{S}_{k}(\sigma)$ informs the total number of possible $k$-periodic orbits in the system. When substituted into $\psi_{k}(x)$, each individual root of $\mathbb{S}_{k}(\sigma)=0$ "projects" $\psi_{k}(x)$ into the $\sigma$ selected individual orbit. Normally, $\mathbb{S}_{k}(\sigma)$ is a reducible polynomial over the integers: nonlinear factors of degree $\partial_{k}$ correspond to orbital clusters, namely to irreducible polynomial aggregates commingling together a total of $\partial_{k}$ orbits. Linear factors correspond to non-clustered single orbits of degree $k$.

For period-four there are three possible orbits, all encoded simultaneously by the doublet:

$$
\begin{aligned}
& \psi_{4}(x)=x^{4}-\sigma x^{3}+\frac{1}{2}\left(\sigma^{2}+\sigma-8\right) x^{2}-\frac{1}{6}\left(\sigma^{3}+3 \sigma^{2}-20 \sigma+2\right) x \\
&+\frac{1}{24}(\sigma-3)\left(\sigma^{3}+9 \sigma^{2}-2 \sigma-16\right) \\
& \mathbb{S}_{4}(\sigma)=(\sigma+1)\left(\sigma^{2}-\sigma-4\right) .
\end{aligned}
$$

Substituting $\sigma=-1$ into $\psi_{4}(x)$ we obtain the orbit $o_{4,1}(x)$, while for $(1-\sqrt{17}) / 2$ and $(1+$ $\sqrt{17}) / 2$, roots of the quadratic factor, we get $o_{4,2}(x)$ and $o_{4,3}(x)$, respectively:

$$
\begin{aligned}
& o_{4,1}(x)=x^{4}+x^{3}-4 x^{2}-4 x+1, \\
& o_{4,2}(x)=x^{4}-\frac{1}{2}(1-\sqrt{17}) x^{3}-\frac{1}{2}(3+\sqrt{17}) x^{2}-(2+\sqrt{17}) x-1, \\
& o_{4,3}(x)=x^{4}-\frac{1}{2}(1+\sqrt{17}) x^{3}-\frac{1}{2}(3-\sqrt{17}) x^{2}-(2-\sqrt{17}) x-1 .
\end{aligned}
$$

When multiplied together, $o_{4,2}(x)$ and $o_{4,3}(x)$ produce the orbital cluster, or aggregate:

$$
c_{4,1}(x)=o_{4,2}(x) \cdot o_{4,3}(x)=x^{8}-x^{7}-7 x^{6}+6 x^{5}+15 x^{4}-10 x^{3}-10 x^{2}+4 x+1,
$$

a cluster that may be obtained directly by eliminating $\sigma$ between $\psi_{4}(x)$ and $\sigma^{2}-\sigma-4$.

Note that the product of $o_{4,2}(x)$ and $o_{4,3}(x)$, which have algebraic coefficients, resulted in a cluster with integer coefficients, a generic characteristic. Technically, $o_{4,2}(x)$ and $o_{4,3}(x)$ are defined by relative quadratic equations of motion ${ }^{9}$. Manifestly, $c_{4,1}(x)$ decomposes over the field $\mathbb{Q}(\sqrt{17})$. The orbit $o_{4,1}(x)$ has always integer coefficients and is always an exact representation for the orbit. In sharp contrast, when projected onto the real axis, $o_{4,2}(x)$ and $o_{4,3}(x)$ will have necessarily approximate numerical coefficients. Thus, the symmetries clearly visible between Eqs. (4) and (5) will be totally obliterated. 
This unambiguous dichotomic distinction between orbits remains valid for other periods and neatly displays the enhanced insight obtained by working with exact equations of motion.

Doublets like Eqs. (1) and (2) may be determined for arbitrary periods. Expressions for arbitrary values of $a$ of the quadratic map and arbitrary $(a, b)$ of the Hénon map are available 2425 .

\subsection{The period five carrier}

For period-five there are six possible orbits, all encoded simultaneously by the doublet:

$$
\begin{aligned}
& \psi_{5}(x)=(\left.360 \sigma^{2}-360 \sigma-240\right) x^{5}-120 \sigma\left(3 \sigma^{2}-3 \sigma-2\right) x^{4}+60\left(\sigma^{2}+\sigma-10\right)\left(3 \sigma^{2}-3 \sigma-2\right) x^{3} \\
&-\left(60 \sigma^{5}+90 \sigma^{4}-1800 \sigma^{3}+1710 \sigma^{2}+1860 \sigma-1200\right) x^{2} \\
&+\left(15 \sigma^{6}+45 \sigma^{5}-735 \sigma^{4}+375 \sigma^{3}+3480 \sigma^{2}-2700 \sigma-1200\right) x \\
&-3 \sigma^{7}-12 \sigma^{6}+192 \sigma^{5}+30 \sigma^{4}-2061 \sigma^{3}+1446 \sigma^{2}+4248 \sigma-3600 \\
& \mathbb{S}_{5}(\sigma)=(\sigma-1)\left(\sigma^{2}+\sigma-8\right)\left(\sigma^{3}-\sigma^{2}-10 \sigma+8\right) .
\end{aligned}
$$

Eliminating $\sigma$ between $\psi_{5}(x)$ and, successively, $\sigma-1, \sigma^{2}+\sigma-8$, and $\sigma^{3}-\sigma^{2}-10 \sigma+8$, we get, apart from multiplicative constants used to eliminate denominators in $\psi_{5}(x)$,

$$
\begin{aligned}
& o_{5,1}(x)=x^{5}-x^{4}-4 x^{3}+3 x^{2}+3 x-1, \\
& c_{5,1}(x)=x^{10}+x^{9}-10 x^{8}-10 x^{7}+34 x^{6}+34 x^{5}-43 x^{4}-43 x^{3}+12 x^{2}+12 x+1, \\
& c_{5,2}(x)=x^{15}-x^{14}-14 x^{13}+13 x^{12}+78 x^{11}-66 x^{10}-220 x^{9}+165 x^{8}+330 x^{7} \\
& \quad-210 x^{6}-252 x^{5}+126 x^{4}+84 x^{3}-28 x^{2}-8 x+1 .
\end{aligned}
$$

The clusters factor into quintics over $\mathbb{Q}(\sqrt{33})$ and $\mathbb{Q}(\sqrt[3]{-62+95 \sqrt{-3}})$, respectively, thereby providing explicit expressions for the remaining five period-five orbits. As before, clustered orbits involve relative quadratic and cubic equations, with algebraic (non-integer) coefficients which, in numerical computations cannot be determined exactly.

\subsection{The period six carrier}

For period-six there are nine possible orbits, all encoded simultaneously by the doublet:

$$
\begin{aligned}
\psi_{6}(x)= & 160 \varphi^{2} \sigma^{2}\left(x^{6}-\sigma x^{5}\right)+80 \sigma^{2}(\sigma+4)(\sigma-3) \varphi^{2} x^{4} \\
& -40 \sigma \varphi\left(2 \sigma^{7}+\sigma^{6}-86 \sigma^{5}+126 \sigma^{4}+358 \sigma^{3}-343 \sigma^{2}-50 \sigma+56\right) x^{3} \\
& +20 \sigma^{2} \varphi\left(\sigma^{7}+3 \sigma^{6}-70 \sigma^{5}+48 \sigma^{4}+679 \sigma^{3}-683 \sigma^{2}-1218 \sigma+1048\right) x^{2} \\
& -4 \sigma \varphi\left(\sigma^{9}+6 \sigma^{8}-91 \sigma^{7}-78 \sigma^{6}+1693 \sigma^{5}-976 \sigma^{4}-6911 \sigma^{3}+5496 \sigma^{2}+2508 \sigma-2128\right) x \\
& \quad+2 \sigma^{14}+14 \sigma^{13}-247 \sigma^{12}-268 \sigma^{11}+7984 \sigma^{10}-8072 \sigma^{9}-80966 \sigma^{8}+157668 \sigma^{7} \\
& \quad+184938 \sigma^{6}-530694 \sigma^{5}+88965 \sigma^{4}+373032 \sigma^{3}-197156 \sigma^{2}-13440 \sigma+15680,
\end{aligned}
$$

where $\varphi \equiv 3 \sigma^{3}-7 \sigma^{2}-13 \sigma+13$. Apart from multiplicative constants used to eliminate denominators in $\psi_{6}(x)$, by selecting $\sigma=1$ and $\sigma=-1$ we get the orbits and discriminants:

$$
\begin{array}{ll}
o_{6,1}(x)=x^{6}-x^{5}-5 x^{4}+4 x^{3}+6 x^{2}-3 x-1, & \Delta_{6,1}=13^{5}=371293, \\
o_{6,2}(x)=x^{6}+x^{5}-6 x^{4}-6 x^{3}+8 x^{2}+8 x+1, & \Delta_{6,2}=3^{3} \cdot 7^{5}=453789 .
\end{array}
$$

Again, for roots of the cubic and quartic factors in Eq. (13), the resulting coefficients in Eq. 12 are more complicated algebraic numbers, not integers. When all orbits arising from the same $\sigma-$ factor are multiplied together one obtains a cluster, a polynomial aggregate with integer coefficients and degree $\partial=m k$, multiple of the period $k$, where $m>1$ is an integer:

$$
\begin{aligned}
c_{6,1}(x)= & x^{18}-18 x^{16}+x^{15}+135 x^{14}-15 x^{13}-546 x^{12}+90 x^{11}+1287 x^{10}-276 x^{9} \\
& -1782 x^{8}+459 x^{7}+1385 x^{6}-405 x^{5}-534 x^{4}+170 x^{3}+72 x^{2}-24 x+1, \\
c_{6,2}(x)= & x^{24}+x^{23}-24 x^{22}-23 x^{21}+252 x^{20}+229 x^{19}-1521 x^{18}-1292 x^{17}+5832 x^{16} \\
& +4540 x^{15}-14822 x^{14}-10282 x^{13}+25284 x^{12}+15001 x^{11}-28667 x^{10}-13653 x^{9}
\end{aligned}
$$


Table 1. The nine period-six orbits $o_{6, j}$ of the map $x_{t+1}=2-x_{t}^{2}$. Here, $\sigma_{6, j}=\sum x_{j}$ is the sum of the orbital points. The triad and quartet of $\sigma_{6, j}$ values are roots of the cubic and quartic factors in Eq. [13), respectively.

\begin{tabular}{|c|c|c|c|c|c|c|c|}
\hline Orbit & $x_{1}$ & $x_{2}$ & $x_{3}$ & $x_{4}$ & $x_{5}$ & $x_{6}$ & $\sigma_{6, j}$ \\
\hline$o_{6,1}$ & -1.770912051306 & -1.1361 & 0.7093 & 1.4969 & -0.2407 & 1.9421 & 1 \\
\hline$o_{6,2}$ & -1.911145611572 & -1.6523 & -0.7301 & 1.4670 & -0.1521 & 1.9769 & -1 \\
\hline$o_{6,3}$ & -1.990061550730 & -1.9605 & -1.8436 & -1.3989 & 0.0431 & 1.9981 & -5.142457360 \\
$o_{6,4}$ & -1.756443146740 & -1.0849 & 0.8230 & 1.3227 & 0.2505 & 1.9372 & 1.491252188 \\
$o_{6,5}$ & -0.912421314706 & 1.1675 & 0.6369 & 1.5944 & -0.5421 & 1.7061 & 3.651205171 \\
\hline$o_{6,6}$ & -1.990663269435 & -1.9629 & -1.8530 & -1.4336 & -0.0552 & 1.9970 & -5.287613777 \\
$o_{6,7}$ & -1.916491658218 & -1.6730 & -0.7989 & 1.3618 & 0.1455 & 1.9788 & -0.902246984 \\
$o_{6,8}$ & -1.559348708126 & -0.4314 & 1.8139 & -1.2902 & 0.3354 & 1.8875 & 0.756484903 \\
$o_{6,9}$ & -0.971966826485 & 1.0553 & 0.8863 & 1.2145 & 0.5250 & 1.7244 & 4.433375858 \\
\hline
\end{tabular}
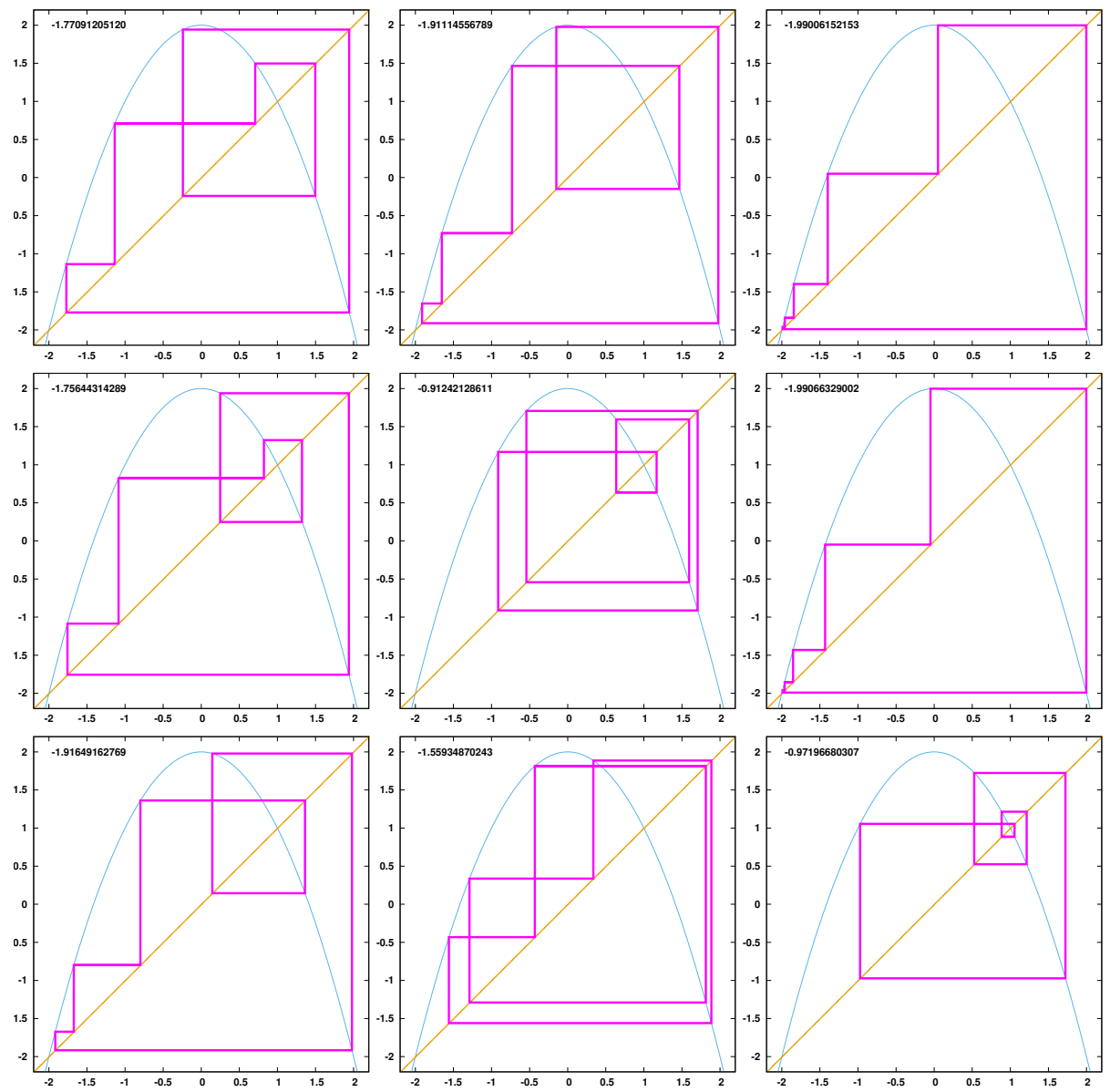

Fig. 1. Return maps $x_{t} \times x_{t+1}$ for the nine period-6 orbits. Numbers refer to the leftmost orbital coordinate. Some of the orbits are topologically identical, despite their very distinct algebraic character.

$$
+20886 x^{8}+7168 x^{7}-9126 x^{6}-1802 x^{5}+2085 x^{4}+101 x^{3}-180 x^{2}+12 x+1 .
$$

Independently from $\psi_{6}(x)$, the Maple driver given in Appendix A exemplifies how to extract $o_{6,1}(x)$, $o_{6,2}(x), c_{6,1}(x)$, and $c_{6,2}(x)$ directly from the quadratic equation of motion.

The orbital points for all nine period-six orbits are collected in Table 1 together with the sums $\sigma_{6, j}$. Return maps for all nine orbits are illustrated in Fig. 1. Numbers inside panels identify the leftmost orbital point. From Fig. 1 one sees that some orbits are topologically identical despite the very distinct 
nature of the algebraic numbers underlying them.

It is interesting to mention that Eqs. 14 and (15) imply a novel twist in the current understanding of polynomial interdependence. As individual orbits, they are obtained as two "projections" arising from a common mathematical origin, the carrier $\psi_{6}(x)$. Therefore, rather than independent orbits, they are in a certain sense a kind of "conjugated" orbits. Furthermore, since all nine orbits arise from the same carrier $\psi_{6}(x)$, the pair of orbits is also conjugated to the remaining seven orbits. This illustrates the existence of a complex and subtle arithmetical interdependence lurking among such orbits, apparently rather different from the usual field isomorphisms familiar from Galois theory of equations. Orbital carriers allow conjugated orbits to have coefficients from very distinct number fields, a concept alien to the standard theory.

As for the remaining polynomials, $o_{6,1}(x)$ factors into a pair of cubics over $\mathbb{Q}(\sqrt{13})$. The cluster $c_{6,2}(x)$ factors into two equations of degree nine over $\mathbb{Q}(\sqrt{21})$. However, these polynomials mix roots of distinct orbits, since their degree is not a multiple of six. For $c_{6,1}(x)$, six cubics are obtained over $\mathbb{Q}(\sqrt[3]{\alpha})$, where $\alpha=-154+42 \sqrt{-3}-18 \sqrt{-7}+30 \sqrt{21}$. For $c_{6,2}(x)$, eight cubics are obtained over $\mathbb{Q}(\sqrt{\beta})$, where $\beta=65-13 \sqrt{5}+15 \sqrt{15}-3 \sqrt{65}$. These factorizations provide explicit and exact solutions for all period-six orbits. Note that the factors of $\mathbb{S}_{k}(\sigma)$ reveal how orbits are distributed into clusters and single orbits, if any.

\section{Orbital inheritance}

A simple example allows one to grasp easily what inheritance means 6778 . To this end, we apply the nonlinear transformation $x^{3}-3 x$ to $o_{6,2}(x)$, obtaining the identity:

$$
c_{6,1}(x)=o_{6,2}\left(x^{3}-3 x\right) .
$$

This identity shows that, as soon as the roots $z_{i}$ of $o_{6,2}(x)=0$ are determined, three new orbits follow from the zeros of the six cubics

$$
x^{3}-3 x-z_{i}=0 .
$$

Therefore, since $o_{6,2}(x)$ factors into a pair of cubics over $\mathbb{Q}(\sqrt{21})$,

$$
\begin{aligned}
o_{6,2}(x)= & \left(x^{3}+\frac{1}{2}(1-\sqrt{21}) x^{2}-\frac{1}{2}(1+\sqrt{21}) x+\frac{1}{2}(5+\sqrt{21})\right) \times \\
& \left(x^{3}+\frac{1}{2}(1+\sqrt{21}) x^{2}-\frac{1}{2}(1-\sqrt{21}) x+\frac{1}{2}(5-\sqrt{21})\right),
\end{aligned}
$$

their roots provide exact analytical solutions in terms of radicals for all orbital points of the 18th-degree cluster $c_{6,1}(x)=0$. Such exact solutions are simple cascades, towers, of relative cubic irrationalities 9 . Incidentally, Maple surprisingly fails to solve the sextic $o_{6,2}(x)$ using aux:=solve $(062, \mathrm{x})$; convert (aux [1],radical); But it correctly breaks $o_{6,2}(x)$ into a pair of cubics when adding input from the sextic discriminant: factor $\left(062,21^{\wedge}(1 / 2)\right)$;

What about the inherent character of the irrationalities underlying period-six orbital point? This question is particularly interesting because, while compositions of relative quadratic irrationalities are

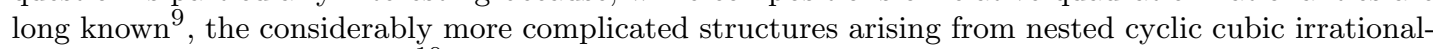
ities remains essentially open 10 . Thus, present day computer algebra systems still have to grapple with difficulties to simplify expressions containing cubic and higher roots 11/12|13|14. By way of illustration, consider to reassemble the cubics in Eq. (20) starting from the exact expressions of their three roots. In this case, we get the leftmost number below as the second coefficient in the topmost equation, not its most simplified version:

$$
\frac{119-21 \sqrt{-3}-27 \sqrt{-7}+31 \sqrt{21}}{-77+21 \sqrt{-3}+9 \sqrt{-7}-15 \sqrt{21}}=-\frac{49+11 \sqrt{21}}{2(14+3 \sqrt{21})}=\frac{1}{2}(1-\sqrt{21}) \simeq-1.791287 .
$$

Similarly garbled expressions are obtained for all other coefficients in Eq. 200. The good news is that such expressions provide clues regarding the subfield structure underlying the solutions. Clues may be also obtained from the algebraic numbers solving the factors in $\mathbb{S}_{k}(\sigma)$.

\section{Inheritance systematics up to periods $k \leq 12$}

\subsection{Periods $k \leq 11$}

Using a slightly adapted version of the ad-hoc Maple driver given in appendix A, we computed systematically all genuine factors defining orbits with period $k \leq 12$. A summary of the relevant data obtained for $k \leq 11$ is given in Table 2. This table reveals a number of interesting facts and trends: 
Table 2. Data summary of polynomial factors as a function of the period $k$. Type refers either to orbits $o_{k, j}$ or orbital clusters $c_{k, j}$, $\partial$ is the degree of the corresponding polynomial, $D=\Delta$ are the standard polynomial and field discriminants, and $L$ is the length, the number of digits of the discriminants. For a given $k$, highlighted cells indicate discriminants arising from identical prime numbers (see text).

\begin{tabular}{|c|c|c|c|c||c|c|c|c|c|}
\hline$k$ & Type & $\partial$ & $D=\Delta$ & $L$ & $k$ & Type & $\partial$ & $D=\Delta$ & $L$ \\
\hline 3 & $\mathbf{o}_{3,1}$ & 3 & $7^{2}$ & 2 & 9 & $\mathbf{o}_{9,1}$ & 9 & $3^{22}$ & 11 \\
& $\mathbf{o}_{3,2}$ & 3 & $9^{2}$ & 2 & & $\mathbf{o}_{9,2}$ & 9 & $19^{8}$ & 11 \\
4 & $\mathbf{o}_{4,1}$ & 4 & $3^{2} \cdot 5^{3}$ & 4 & & $c_{9,1}$ & 18 & $3^{9} \cdot 19^{17}$ & 27 \\
& $c_{4,1}$ & 8 & $17^{7}$ & 9 & & $c_{9,2}$ & 36 & $73^{35}$ & 66 \\
5 & $\mathbf{o}_{5,1}$ & 5 & $11^{4}$ & 5 & & $c_{9,3}$ & 54 & $3^{81} \cdot 19^{51}$ & 104 \\
& $c_{5,2}$ & 10 & $3^{5} \cdot 11^{9}$ & 12 & & $c_{9,4}$ & 162 & $3^{405} \cdot 19^{153}$ & 389 \\
& $c_{5,3}$ & 15 & $31^{14}$ & 21 & & $c_{9,5}$ & 216 & $7^{180} \cdot 73^{213}$ & 550 \\
\hline 6 & $\mathbf{o}_{6,1}$ & 6 & $13^{5}$ & 6 & 10 & $\mathbf{o}_{10,1}$ & 10 & $5^{17}$ & 12 \\
& $\mathbf{o}_{6,2}$ & 6 & $3^{3} \cdot 7^{5}$ & 6 & & $c_{10,1}$ & 20 & $41^{19}$ & 31 \\
& $c_{6,1}$ & 18 & $3^{27} \cdot 7^{15}$ & 26 & & $c_{10,2}$ & 30 & $3^{15} \cdot 31^{29}$ & 51 \\
& $c_{6,2}$ & 24 & $5^{18} \cdot 13^{22}$ & 38 & & $c_{10,3}$ & 80 & $5^{60} \cdot 41^{78}$ & 168 \\
7 & $c_{7,1}$ & 21 & $43^{20}$ & 33 & & $c_{10,4}$ & 150 & $11^{135} \cdot 31^{145}$ & 357 \\
& $c_{7,2}$ & 42 & $3^{21} \cdot 43^{41}$ & 77 & & $c_{10,5}$ & 300 & $3^{150} \cdot 11^{270} \cdot 31^{290}$ & 786 \\
& $c_{7,3}$ & 63 & $127^{62}$ & 131 & & $c_{10,6}$ & 400 & $5^{700} \cdot 41^{390}$ & 1119 \\
\hline 8 & $c_{8,1}$ & 16 & $3^{8} \cdot 17^{15}$ & 23 & 11 & $\mathbf{o}_{11,1}$ & 11 & $23^{10}$ & 14 \\
& $c_{8,2}$ & 32 & $5^{24} \cdot 17^{30}$ & 54 & & $c_{11,1}$ & 44 & $89^{43}$ & 84 \\
& $c_{8,3}$ & 64 & $3^{32} \cdot 5^{48} \cdot 17^{60}$ & 123 & & $c_{11,3}$ & 341 & $683^{340}$ & 964 \\
& $c_{8,4}$ & 128 & $257^{127}$ & 307 & & $c_{11,4}$ & 682 & $3^{341} \cdot 683^{681}$ & 2093 \\
& & & & & & $c_{11,5}$ & 968 & $23^{924} \cdot 89^{957}$ & 3124 \\
\hline
\end{tabular}

(1) The growth of the number of single orbits is much smaller than cluster growth.

(2) Periods $k=7$ and $k=8$ contain only orbital clusters, no single orbits.

(3) Orbits and clusters are all monogenic, i.e. the discriminant $D$ of their minimal polynomial coincides with their field discriminant $\Delta$. Therefore, orbits and clusters admit power integral bases. For details, see Ref. 26 .

(4) The degree of single orbits and clusters is always a multiple of the period $k$.

(5) As indicated by the length $L$ giving the number of digits in the discriminants, $D$ and $\Delta$ grow fast with the period. However, they contain powers of relatively small prime numbers.

(6) The discriminants of, e.g., $c_{11,5}$ contain 3124 digits. It would be computationally hard to factor it if it was not a simple product of powers of a few identical and small primes, 23 and 89.

(7) The highlighted values of $D=\Delta$ for $k=6,9$, and 10 summarize all cases of inheritance found for $k \leq 11$.

(8) For $k=6$ the ratio of the polynomial degrees are $\partial\left(c_{6,1}\right) / \partial\left(o_{6,1}\right)=3$. Similarly, for $k=9$ the ratios are $\partial\left(c_{9,4}\right) / \partial\left(c_{9,3}\right)=\partial\left(c_{9,3}\right) / \partial\left(c_{9,1}\right)=3$. Inheritance among these orbits involves the aforementioned cubic transformation: $c_{9,3}(x) \equiv c_{9,1}\left(x^{3}-3 x\right)$ and $c_{9,4}(x) \equiv c_{9,3}\left(x^{3}-3 x\right)$.

(9) In contrast, for $k=10$ the ratio is $\partial\left(c_{10,6}\right) / \partial\left(o_{10,3}\right)=5$, implying inheritance involving a quintic nonlinear transformation 7 . In this case, we have $c_{10,6}(x) \equiv o_{10,3}\left(x^{5}-5 x^{3}+5 x\right)$.

(10) For a given period $k$, the discriminants $D$ and $\Delta$ involve certain combinations of a small set of primes. We were not able to find interconnections between orbits with discriminants arising from powers of distinct primes, although we see no reason to rule out the possibility of intricate interconnections yet to be discovered.

(11) From Table 2 it seems reasonable to conjecture inheritance to exist among polynomials with discriminants composed by powers of the same primes.

\subsection{Period $k=12$}

Table 3 summarizes data obtained for the sixteen individual factors resulting from the computation and factorization of the 4020th degree polynomial which contains all genuine period twelve orbits and clusters. These factors corroborate the properties listed above for $k \leq 11$. Note the fast increase in the number of digits of the discriminants, which for $c_{12,16}(x)$ contains no less than 6770 digits. In order to factor arbitrary numbers of this size, computers need to check numbers of the order of the size of the square-root of the number to be factored, in the present case roughly $10^{3385}$. 
Table 3. Individual factors of the 4020th degree polynomial containing all period twelve orbits and orbital clusters. Here, $\partial$ refers to the degree of individual factors, while length is the number of digits contained in the discriminants $D=\Delta$. Similar highlighting is used for discriminants defined by identical prime numbers. No more than pairs of interdependent orbits are observed.

\begin{tabular}{|c|c|c|c|}
\hline$\ell$ & Degree $\partial$ & $D=\Delta$ & Length \\
\hline 1 & 12 & $5^{9} \cdot 7^{10}$ & 15 \\
2 & 12 & $3^{18} \cdot 5^{9}$ & 15 \\
3 & 12 & $3^{6} \cdot 13^{11}$ & 16 \\
4 & 24 & $3^{12} \cdot 5^{18} \cdot 7^{20}$ & 36 \\
5 & 36 & $3^{54} \cdot 13^{33}$ & 63 \\
6 & 36 & $7^{30} \cdot 13^{33}$ & 63 \\
7 & 48 & $3^{24} \cdot 5^{36} \cdot 13^{44}$ & 86 \\
8 & 72 & $3^{108} \cdot 5^{54} \cdot 7^{60}$ & 140 \\
9 & 72 & $3^{36} \cdot 7^{60} \cdot 13^{66}$ & 142 \\
10 & 120 & $241^{119}$ & 284 \\
11 & 144 & $5^{108} \cdot 7^{120} \cdot 13^{132}$ & 324 \\
12 & 144 & $3^{216} \cdot 5^{108} \cdot 13^{132}$ & 326 \\
13 & 216 & $3^{324} \cdot 7^{180} \cdot 13^{198}$ & 528 \\
14 & 288 & $3^{144} \cdot 5^{216} \cdot 7^{240} \cdot 13^{264}$ & 717 \\
15 & 864 & $3^{1296} \cdot 5^{648} \cdot 7^{720} \cdot 13^{792}$ & 2562 \\
16 & 1920 & $17^{1800} \cdot 241^{1912}$ & 6770 \\
\hline \hline
\end{tabular}

Table 4. Growth of the number $N_{k}$ of periodic orbits, as a function of the period $k$. The number of orbits roughly doubles as $k$ increases. For simple equations and Maple implementations to obtain arbitrary values of $N_{k}$ see Refs. ${ }^{27,28}$.

\begin{tabular}{|c||c|c|c|c|c|c|c|c|c|}
\hline$k$ & 12 & 13 & 14 & 15 & 16 & 17 & 18 & 19 & 20 \\
\hline$N_{k}$ & 335 & 630 & 1161 & 2182 & 4080 & 7710 & 14532 & 27594 & 52377 \\
$N_{k} / N_{k-1}$ & 1.80 & 1.88 & 1.84 & 1.88 & 1.87 & 1.90 & 1.88 & 1.90 & 1.90 \\
\hline
\end{tabular}

Numbers with 6770 digits are well beyond the capabilities of factorization, and also well beyond the numbers currently used in data encryption. For instance, consider that the lifetime of the universe, currently estimated to be some 13.8 billion years, roughly $10^{18}$ seconds, a number with 19 digits. Assuming a computer able to test one million factorizations per second, during the lifetime of the universe it would be able to check some $10^{24}$ possibilities. However, for 6770 digits, roughly $10^{6770}$, one would need to check $10^{3385}$ possibilities, meaning that the time to do this amounts to roughly $10^{3385-24}=10^{3361}$ times the lifetime of the universe! Fortunately, however, the very big numbers in Table 3 involve products of just a few and small primes, allowing them to be factored, as indicated in the Table. The passage here is exceedingly narrow. Slight changes in the coefficients may preclude factorization.

The most conspicuous difference when comparing the numbers in Table 3 with analogous results for the lower periods in Table 2 is the surprising increase of the number of polynomials displaying inheritance. For instance, abbreviating $X=x^{3}-3 x$, we find the following five nonlinear interconnections among polynomials of quite high degrees: $c_{12,5}(x) \equiv o_{12,3}(X), c_{12,8}(x) \equiv c_{12,4}(X)$, $c_{12,12}(x) \equiv c_{12,7}(X), c_{12,13}(x) \equiv c_{12,9}(X)$, and $c_{12,15}(x) \equiv c_{12,14}(X)$. The verification of these identities requires ad-hoc handling because of recurring Maple warnings "stack limit reached".

Table 4 illustrates how fast the number of orbits grows as a function of the period $k$. A simple and explicit formula and its Maple implementation to compute such growth is available in the literature $\underline{27 \mid 28}$. It would be interesting to extend the present calculations and check inheritance for the promising cases $k=14,15,16$ and 18, something that should be feasible already by someone with access to more powerful resources than available to us.

Two additional aspects are worth mentioning: First, periodic orbits may be found by studying preperiodic points ${ }^{8}$. Such procedure involves just straightforward but somewhat tedious computations, due to the large number of factors and orbits involved. Fortunately, the procedure involving preperiodic points may be programmed to run automatically. Second, by a process of reverse engineering and by 
suitably summing orbital points, one may recover the several individual factors arising in the $\mathbb{S}_{k}(\sigma)$ polynomials. For instance, in Appendix B we compute explicitly the three factors composing $\mathbb{S}_{7}(\sigma)$. For single orbits the factors are very simple to find. For instance, the single period-twelve orbits are

$$
\begin{gathered}
o_{12,1}(x)=x^{12}+x^{11}-12 x^{10}-11 x^{9}+54 x^{8}+43 x^{7}-113 x^{6}-71 x^{5} \\
+110 x^{4}+46 x^{3}-40 x^{2}-8 x+1, \\
o_{12,2}(x)=x^{12}-12 x^{10}+x^{9}+54 x^{8}-9 x^{7}-112 x^{6}+27 x^{5}+105 x^{4} \\
-31 x^{3}-36 x^{2}+12 x+1, \\
o_{12,3}(x)=x^{12}+x^{11}-12 x^{10}-12 x^{9}+53 x^{8}+53 x^{7}-103 x^{6}-103 x^{5} \\
+79 x^{4}+79 x^{3}-12 x^{2}-12 x+1,
\end{gathered}
$$

and we immediately recognize that $s(s+1)^{2}$ are the linear factors of $\mathbb{S}_{12}(\sigma)$, a curious degenerate multiplicity situation which seems to foretell that $\psi_{12}(x)$ will be a reducible polynomial. Analogously, linear factors of $\mathbb{S}_{k}(\sigma)$ may be read directly from the coefficients of the orbits:

$$
\begin{aligned}
o_{9,1}(x) & =x^{9}-9 x^{7}+27 x^{5}-30 x^{3}+9 x-1, \\
o_{9,2}(x) & =x^{9}-x^{8}-8 x^{7}+7 x^{6}+21 x^{5}-15 x^{4}-20 x^{3}+10 x^{2}+5 x-1, \\
o_{10,1}(x) & =x^{10}-10 x^{8}+35 x^{6}-x^{5}-50 x^{4}+5 x^{3}+25 x^{2}-5 x-1, \\
o_{11,1}(x) & =x^{11}-x^{10}-10 x^{9}+9 x^{8}+36 x^{7}-28 x^{6}-56 x^{5}+35 x^{4}+35 x^{3}-15 x^{2}-6 x+1 .
\end{aligned}
$$

It is quite challenging to decompose orbital clusters combining more than two orbits, particularly those combining an odd number of orbits. However, the coefficients of such decompositions hide the secretest truth and most interesting relations among numbers which fix orbital individuality.

\section{Conclusions and outlook}

This paper presented explicit expressions for orbital carriers of periods 4,5 , and 6 . In addition, the systematics of orbital inheritance was considered for all periods $k \leq 12$. Evidence was found that inheritance becomes more abundant as the period increases. Useful insight was obtained from the exact properties of equations of motion, instead of orbital points. An interesting open challenge is to compute the distinct factors arising for orbits of periods $k=14,15,16$ and 18, and to check if they also display inheritance and relations with orbits of lower periods, if any. A much harder problem seems to be to find out if orbits not displaying inheritance may nevertheless display some other type of interdependence. If found, this would certainly reveal unanticipated interconnections among families of algebraic numbers.

As it is visible from Tables 2 and 3 the growth of the polynomial degrees $\partial_{k}$ as a function of $k$ and their partition into proper divisors of $k$ are interesting open combinatorial questions. What is the mechanism behind the decomposition of the number $N_{k}$ of periodic orbits into the several degrees $\partial_{k}$ of the polynomial set defining $k$-periodic orbits? For instance, the 4020th degree polynomial of period-12 orbits is partitioned into sixteen factors recorded in Table 3 . What would be, say, the corresponding partition for the 16254th degree polynomial corresponding to period-14 orbits and clusters? Or the 32730 th degree polynomial for period-15? Or the 65280 th degree polynomial for period-16? Note that the partitions listed in Table 2 are not unique: for $k=6$, instead of $6+6+18+24$, we could equally well have $12+18+24,12+12+30$, etc. Such alternative partitions, however, are never observed in the present context. It is clear that the partition sets have many elements, and an interesting combinatorial challenge is to count them all and to predict partitions that may be observed for a given period of a given map.

Finally, for applications in physics and dynamical systems, it is of interest to mention that in algebraic number theory one knows that every cyclotomic field is an Abelian extension of the rational numbers $\mathbb{Q}$. In this context, an important discovery is the so-called Kronecker-Weber theorem, stating that every finite Abelian extension of $\mathbb{Q}$ can be generated by roots of unity, i.e. Abelian extensions are contained within some cyclotomic field. Equivalently, every algebraic integer whose Galois group is Abelian can be expressed as a sum of roots of unity with rational coefficients. For details see, e.g., Edwards 29 . The study of the partition generating limit of the quadratic map $x_{t+1}=a-x_{t}^{2}$ seems to 
lend hope that for $a=2$ the map may also share an analogous correspondence with Abelian equations as the one embodied in the Kronecker-Weber theorem 30 , which is intrinsically related to the cyclotomic polynomials generated by the map when $a=0$, whose dynamics, unbeknownst to him, was studied by Gauss in Sectio Septima of his Disquisitiones Arithmeticæ. Such enticing possibility of correspondence deserves to be further investigated.

\section{Acknowledgments}

This work was started during a visit to the Max-Planck Institute for the Physics of Complex Systems, Dresden, gratefully supported by an Advanced Study Group on Forecasting with Lyapunov vectors. The author was partially supported by CNPq, Brazil, grant 304719/2015-3.

\section{Appendix A. Maple driver to generate period six orbits and clusters}

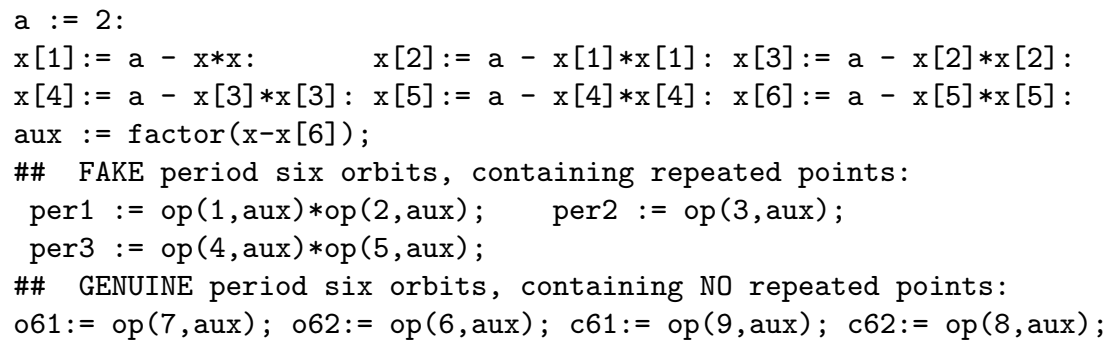

The above assignments are correct under Maple 2014, but are easy to adjust if emerging differently. Manifestly, the driver above may be easily adapted to generate equations for other periods.

Appendix B. Determination of the three factors composing $\mathbb{S}_{\mathbf{7}}(\sigma)$

Here, in contrast to the arithmetic work done so far, we resort to numerically computed orbital points to illustrate how to find exact representations for the individual factors composing $\mathbb{S}_{7}(\sigma)$. The three clusters whose roots give all period-seven orbital points may be easily generated by slightly adapting the Maple driver given in Appendix A. Such clusters read as follows:

$$
\begin{aligned}
& c_{7,1}(x)=x^{21}-x^{20}-20 x^{19}+19 x^{18}+171 x^{17}-153 x^{16}-816 x^{15}+680 x^{14} \\
&+2380 x^{13}-1820 x^{12}-4368 x^{11}+3003 x^{10}+5005 x^{9}-3003 x^{8} \\
&-3432 x^{7}+1716 x^{6}+1287 x^{5}-495 x^{4}-220 x^{3}+55 x^{2}+11 x-1, \\
& c_{7,2}(x)=x^{42}+x^{41}-42 x^{40}-42 x^{39}+\cdots-3267 x^{4}-3267 x^{3}+44 x^{2}+44 x+1, \\
& c_{7,3}(x)=x^{63}-x^{62}-62 x^{61}+61 x^{60}+\cdots+40920 x^{4}+5456 x^{3}-496 x^{2}-32 x+1 .
\end{aligned}
$$

From them, we extract the $(21+42+63) / 7=18$ orbits summarized in Table 5 After rounding off the real coefficients in the products below, we easily get the exact representations of the three factors composing $\mathbb{S}_{7}(\sigma)$, all with degree multiple of three:

$$
\begin{aligned}
& \prod_{j=1}^{3}\left(\sigma-\sigma_{7, j}\right)=\sigma^{3}-\sigma^{2}-14 \sigma-8 \\
& \prod_{j=4}^{9}\left(\sigma-\sigma_{7, j}\right)=\sigma^{6}+\sigma^{5}-39 \sigma^{4}+63 \sigma^{3}+110 \sigma^{2}-136 \sigma-128 \\
& \prod_{j=10}^{18}\left(\sigma-\sigma_{7, j}\right)=\sigma^{9}-\sigma^{8}-56 \sigma^{7}+118 \sigma^{6}+573 \sigma^{5}-1249 \sigma^{4}-1582 \sigma^{3}+2700 \sigma^{2}+1576 \sigma-32 .
\end{aligned}
$$

Even though an expression for the period-seven carrier pair is still unknown, we were nevertheless able to extract $\mathbb{S}_{7}(\sigma)$. Its factors corroborate the three aggregates $c_{7, m}(x), m=1,2,3$ and identify the relative algebraic nature of the coefficients of individual orbits. Manifestly, the above procedure is valid generically and may be applied to higher periods. Separation of orbits into three groups in Table 5 was only possible due to the a priori knowledge of the three "brute-force factors" in Table 
Table 5. The eighteen period-seven orbits, characterized by one orbital point and the $\operatorname{sum} \sigma_{7, j}$ of all points. The remaining orbital points follow by iterating $x_{t+1}=2-x_{t}^{2}$.

\begin{tabular}{|c||c|c|}
\hline Orbit & $x_{1}$ & $\sigma_{7, j}$ \\
\hline$o_{7,1}$ & -1.97868673615022039558470712622 & -2.88823600884341144649527347953 \\
$o_{7,2}$ & -1.81089647498629323144358511206 & -0.61507162581156506493032243917 \\
$o_{7,3}$ & -1.04188068097586057235256289907 & 4.50330763465497651142559591867 \\
\hline$o_{7,4}$ & -1.99762811048164609235032811636 & -7.24813219626988235042435866316 \\
$o_{7,5}$ & -1.88487616566742883844738056630 & -1.22906022702843317616182868886 \\
$o_{7,6}$ & -1.94098358831481064644663464031 & -0.774908002370389501560130448853 \\
$o_{7,7}$ & -1.61228898351072554484668557445 & 1.84413185283999824109215112803 \\
$o_{7,8}$ & -1.71974598668362014848475373830 & 2.74482456161490583899876274448 \\
$o_{7,9}$ & -1.20298163000374078956875931348 & 3.66314401121380094805540392837 \\
\hline$o_{7,10}$ & -1.99755283242852256525640526121 & -7.17543506383968793122213507689 \\
$o_{7,11}$ & -1.97801140897626144475812107452 & -2.93599431271533079530605723303 \\
$o_{7,12}$ & -1.88125825920768775450635179933 & -1.60237082080316266127006810916 \\
$o_{7,13}$ & -1.93911972959649314295081201674 & -0.525572883742883886011679753072 \\
$o_{7,14}$ & -1.80499303815485256128035783817 & 0.0196507480462068262052865602836 \\
$o_{7,15}$ & -1.60040839696003410107405022267 & 2.19795804752238429773060428492 \\
$o_{7,16}$ & -1.71107014481703192827696436581 & 2.36473479389711276652833789748 \\
$o_{7,17}$ & -1.17956942634103896113267847206 & 3.29077832666324426013918210422 \\
$o_{7,18}$ & -1.01424772773954618184418426842 & 5.36625116497211712320652932523 \\
\hline
\end{tabular}

2 and given explicitly above, in $c_{7, m}(x)$. However, using preperiodic points generated by an infinite family $Q_{\ell}(x)$ of polynomials $\sqrt{8}$, the same three groups my be discovered independently, directly from numerically approximated orbital equations. How to accomplish this will be presented in a forthcoming publication.

\section{References}

1. J. Argyris, G. Faust, M. Haase, and R. Friedrich, An Exploration of Dynamical Systems and Chaos, Second Edition (Springer, Berlin, 2015).

2. M. Cencini, F. Cecconi, and A. Vulpiani, Chaos - From Simple Models to Complex Systems (World Scientific, Singapore, 2010).

3. M. Ausloos, M. Dirickx (eds.), The Logistic Map: Map and the Route to Chaos: From the Beginning to Modern Applications, Proceedings of the "Verhulst 200 on Chaos", Brussels, Belgium (Springer, Heidelberg, 2005).

4. J.A.C. Gallas, Units: remarkable points in dynamical systems, Physica A 222, 125-151 (1995).

5. J.A.C. Gallas, Lasers, stability, and numbers, Physica Scripta 94, 014003 (2019).

6. J.A.C. Gallas, Nonlinear dependencies between sets of periodic orbits, Europhys. Lett. 47, 649-655 (1999).

7. J.A.C. Gallas, Infinite hierarchies of nonlinearly dependent periodic orbits, Phys. Rev. E 63, 016216 (2000).

8. J.A.C. Gallas, Method for extracting arbitrarily large orbital equations of the Pincherle map, Results in Physics 6, 561-567 (2016).

9. J. Sommer, Vorlesungen über Zahlentheorie (Teubner, Leipzig, 1907).

10. B.N. Delone and D.K. Faddeev, The Theory of Irrationalities of the Third Degree (American Mathematical Society, Providence, 1964).

11. S. Landau, How to tangle with a nested radical, Math. Intelligencer 16, 49-55 (1994).

12. S. Landau, Computations with Algebraic Numbers, in J. Grabmeier, E. Kaltofen, and V. Weispfenning (eds.), Computer Algebra Handbook (Springer, Berlin, 2003), pp. 18-19.

13. B.C. Berndt, H.H. Chan, and L.C. Zhang, Ramanujan's association with radicals in India, Am. Math. Month. 104, 905-911 (1997).

14. B.C. Berndt, H.H. Chan, and L.C. Zhang, Radicals and units in Ramanujan's work, Acta Arithm. 87, 145-158 (1998).

15. F. Haake, Quantum Signatures of Chaos, third edition (Springer, Berlin, 2010).

16. J. Marklow, Arithmetic quantum chaos, in Encyclopedia of Mathematical Physics, vol. 1, edited by J.P. Françoise, G.L. Naber, and T.S. Tsun (Academic Press/Elsevier, Oxford, 2006), pp. 212-220. 
17. P. Bleher, Trace formula for quantum integrable systems, lattice-point problem, and small divisors, in Emerging Applications of Number Theory, IMA Vol. Math. Appl. 109 (Springer, New York, 1999), pp. 1-38.

18. A.M.O. de Almeida, C.H. Lewenkopf, and S. Tomsovic, On resumming periodic orbits in the spectra of integrable systems, J. Phys. A 35, 10629-10642 (2002).

19. D. Braun, Dissipative Quantum Chaos and Decoherence (Springer, Berlin, 2001).

20. H. Schomerus and F. Haake, Semiclassical spectra from periodic-orbit clusters in a mixed phase space, Phys. Rev. Lett. 79, 1022-1025 (1997).

21. M. Sieber and K. Richter, Correlations between periodic orbits and their role in spectral statistics, Physica Scripta T-90, 128-133 (2001).

22. J. Li and S. Tomsovic, Exact relations between homoclinic and periodic orbit actions in chaotic systems, Phys. Rev. E 97, 022216 (2018).

23. R.P. Stanley, Enumerative Combinatorics 2, (Cambridge University Press, Cambridge, 1999).

24. A. Endler and J.A.C. Gallas, Arithmetical signatures of the dynamics of the Hénon map, Phys. Rev. E 65, 036231 (2002).

25. A. Endler and J.A.C. Gallas, Existence and characterization of stable ghost orbits in the Hénon map, Physica A 344, 491-497 (2004).

26. J.A.C. Gallas, Monogenic period equations are cyclotomic polynomials, Int. J. Mod. Phys. C 31, 2050058 (2020). Open access: https://arxiv.org/abs/2002.04445

27. J.A.C. Gallas, Counting orbits in conjugacy classes of the Hénon Hamiltonian repeller, Phys. Lett. A 360, 512-514 (2007).

28. O.J. Brison and J.A.C. Gallas, What is the effective impact of the explosive orbital growth in discrete-time one-dimensional polynomial dynamical systems? Physica A 410, 313-318 (2014).

29. H.M. Edwards, Roots of solvable polynomials of prime degree, Expo. Math. 32, 79-91 (2014).

30. N. Schappacher, On the history of Hilbert's twelfth problem, a comedy of errors. In Matériaux pour l'Histoire des Mathématiques au XX $X^{e}$ Siècle. Actes du colloque à la mémoire de Jean Dieudonné. Nice 1996, pp. 243-273. Séminaires et Congrès 3. Paris, Societé Mathématique de France. 